\title{
In memoriam: Professor Minoru Nomura (1930-2016)
}

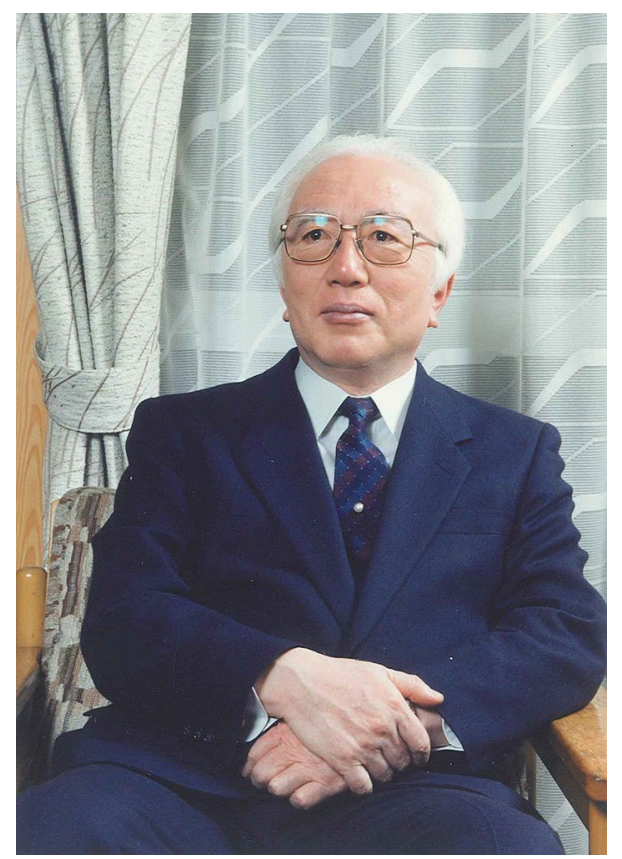

Professor Minoru Nomura, an Honorary Member of the Japanese Society of Fisheries Science, and Professor Emeritus and former President of Tokyo University of Fisheries (currently Tokyo University of Marine Science and Technology), passed away at the age of 85 on 30 March 2016.

Professor Nomura was born in Kanagawa on 3 May 1930. He graduated from the Daiichi Imperial Fisheries Institute in March 1952, and shortly after was hired as a research assistant at Tokyo University of Fisheries. He subsequently held consecutive positions as Assistant Professor (1962-1966), Associate Professor (1966-1968), and Professor (1968-1985) in the Department of Aquaculture, Faculty of Fisheries. During his tenure, he also held various administrative positions in the university including Vice
Dean of the Faculty, Department Chairperson, Director of the Field Research Center at Oizumi, and, later on, President between 1985 and 1991. He played a fundamental role in adding the doctoral course to its Graduate School in 1987 by persistently stressing, to society and governmental agencies, the importance of top-level, specialized education in fisheries science. For his achievements and contributions to the university, he was awarded the title of Professor Emeritus in 1991.

$\mathrm{He}$ dedicated his academic career to the advancement of research and teaching in the field of aquaculture, and made significant contributions to the development of fish cultivation methods. His main work was on the reproduction of salmonids, clarifying the physiological and methodological reasons for the low fertilization and development rates prevalent in hatcheries at that time. His studies led to important discoveries on gonadal development, onset of puberty, and gamete maturation, and on how these processes are influenced by environmental factors. He showed that selective breeding and environmental manipulation of broodstock fish could be used to control the timing of reproduction, enabling the spawning period of trout to be brought forward by more than 1 month. He also published a series of articles reporting on the impairment of sperm motility and fertilization in the presence of damaged roe, the development of a method to wash the eggs with isotonic solutions prior to insemination, and on methods to extend sperm viability for storage. His studies led to great improvements in the production efficiency and quality of artificially produced seeds of trout, with fertilization rates nearly doubling from about $50 \%$ to $90 \%$ as a result. For this work, Professor Nomura was awarded the Japanese Society of Fisheries Science Award in 1967. Due to his dedication to research, his knowledge and warm personality, a deep bond was developed between him and his students, 
which inspired them to do their best and to play leading roles in society. His academic and research achievements were further recognized in 1991 when he was awarded the Japanese Society of Fisheries Science Award of Merit.

In addition to academia, Professor Nomura engaged in multiple professional activities including serving on the Board of Directors and as Vice President of the Japanese Society of Fisheries Science, Vice President of the Asian Fisheries Society, President of the New Fish Development Association, Special Board Member of The Council for Science and Technology of Japan and the Japan National
Commission for UNESCO, among others. In recognition of his outstanding lifetime contributions to science and education he received the Order of the Sacred Treasure, Gold Rays with Neck Ribbon in 1996.

We deeply regret the passing of Professor Nomura and would like to convey our sincere condolences to his family.

Toshio Takeuchi

President, Tokyo University of Marine Science and Technology 